\title{
Análisis de los costes de prevención en obras de construcción
}

\section{Analysis the cost of prevention in construction sites}

\author{
M. López-Alonso ${ }^{(*)}$, P. Ibarrondo-Dávila ${ }^{(*)}, \mathrm{M}^{\mathrm{a}}$ C. Rubio-Gámez ${ }^{(*)}$
}

\section{RESUMEN}

Los costes asociados a la seguridad y salud laboral han sido ampliamente estudiados en diversas actividades industriales. Sin embargo, los modelos tradicionales aplicados para su estudio no resultan efectivos en las obras de construcción. Por otra parte, considerando que son las obras de construcción las que registran las mayores tasas de siniestralidad laboral, y que los accidentes llevan asociados importantes costes, no solo para la sociedad sino también para las empresas en los que ocurren, el objetivo de esta investigación se ha centrado en analizar el alcance que presenta el sistema de gestión de los costes de seguridad en las empresas de construcción. Para ello, se ha llevado a cabo un estudio empírico, al objeto de analizar las posibles relaciones existentes entre el coste de prevención y el coste de los accidentes ocasionado en estas empresas, a partir de una muestra de 40 obras de construcción, en las que se han producido un total de 178 accidentes. En dicho estudio se ha puesto de manifiesto que en las fases de obra de mayor riesgo identificadas, estructuras y movimiento de tierras, se asumen los mayores costes de prevención, a pesar de que también se obtienen los peores datos de siniestralidad.

Palabras clave: Costes; prevención; obras de construcción; seguridad y salud.

\section{ABSTRACT}

The costs associated with work accidents have been thoroughly studied in a variety of activities. However, the traditional models for studying the costs derived from work accidents are not effective for use in analyzing construction sites. Moreover, considering that construction sites are the ones that register the highest number of work accidents and that these accidents have important associated costs, not just for the society but also for companies in which they occur, the main objective of this research is to analyze the health and safety costs management at the construction companies, for this the influence of the construction companies health and safety investment on the costs in which the construction sites incur as a consequence of the work accidents or improper health and safety measures, will be checked. For this reason a sample of 40 construction sites have been analized, with a total of 178 accidents.

Keywords: Costs; prevention; construction sites; health and safety.

(*) Universidad de Granada, (España).

Persona de contacto/Corresponding author: mlopeza@ugr.es (M. López-Alonso)

Cómo citar este artículo/Citation: Lópèz-Alonso, M., Ibarrondo-Dávila, P., Rubio-Gámez, Ma C. (2015). Análisis de los costes de prevención en obras de construcción. Informes de la Construcción, 67(537): e055, doi: http://dx.doi.org/10.3989/ic.13.062.

Licencia / License: Salvo indicación contraria, todos los contenidos de la edición electrónica de Informes de la Construcción se distribuyen bajo una licencia de uso y distribución Creative Commons Reconocimiento no Comercial 3.o. España (cc-by-nc). 


\section{INTRODUCCIÓN Y OBJETIVOS}

El sector de la construcción registra unas tasas de siniestralidad laboral muy elevadas en relación con los Índices de otros sectores de actividad (1) (2) (3) y, como consecuencia, unos altos costes asociados a los accidentes de trabajo. Estos costes son de diversa naturaleza, humanos (que no son directamente cuantificables) y económicos, tanto para las empresas del sector, como para la sociedad en su conjunto (bajas laborales, tratamientos médicos...) (4). Existen paralelamente otros costes como retrasos en los plazos de ejecución, pérdida de imagen o de mercado (5) (6).

Diversos autores han analizado la vertiente económica de la seguridad y salud en el ámbito empresarial y algunos de ellos ponen de manifiesto que los empresarios no consideran que invertir en seguridad sea rentable económicamente, más concretamente consideran que los costes asociados a la siniestralidad laboral no son tan elevados como para que el empresario invierta en seguridad y salud con el único objetivo de evitar dichos costes (7) (8).

Por otra parte, los modelos de análisis y cálculo de costes de prevención a utilizar en las obras de construcción no son fáciles de aplicar en este ámbito por varios motivos. En primer lugar, porque tienen su origen en experiencias realizadas en el sector industrial, de características diferentes a las del sector de la construcción (9). Por otro lado, como señala Andreoni (8), los modelos diseñados tradicionalmente para el análisis de estos costes, se han limitado a una identificación y clasificación de los costes asociados post accidente, en pocas ocasiones tienen en cuenta otros costes relacionados con la seguridad y salud, como los costes de prevención, los costes de formación de los trabajadores o los costes de las visitas de técnicos de las administraciones para la mejora de las condiciones de trabajo y, por último, de acuerdo con Leopold (10) y Rikhardsson (11), cada empresa debería adaptar dichos modelos a sus circunstancias particulares.

En el trabajo de campo desarrollado en esta investigación, la recopilación de algunos datos económicos relacionados con la seguridad y salud y de siniestralidad reflejan las anteriores dificultades. Se trata pues, como objetivo principal en esta investigación, de analizar la influencia que tiene para una obra de construcción la inversión que realiza en medidas en seguridad y salud en fase de ejecución de una obra, sobre los costes en los que la obra podría incurrir como consecuencia de la siniestralidad laboral o de las deficiencias en materia de seguridad y salud que en ella se generaran.

En este sentido, la información interna sobre el coste de los accidentes generados durante la ejecución de la obra de construcción, así como las fases en las que se producen, podría ayudar en la toma de decisiones en materia de seguridad. De acuerdo con Arévalo (12) conocer la repercusión económica de los accidentes, desde la fase de diseño y de sus medidas de prevención, permitiría delimitar un sistema de gestión de la seguridad en la empresa más eficiente desde el origen.

\section{LOS COSTES DE PREVENCIÓN DE RIESGOS LABORALES}

Para el estudio de los costes de seguridad en materia de prevención de riesgos laborales, es necesario introducir los siguientes conceptos:

\subsection{Presupuesto de plan de Seguridad y Salud}

En una obra de construcción el presupuesto destinado a la prevención de riesgos laborales en fase de ejecución representa la valoración económica a priori de las medidas preventivas que se reflejan en el Plan de Seguridad y Salud (en adelante PSS) que realiza el contratista en función del Estudio de Seguridad y Salud (en adelante ESS). En él se analizan, estudian, desarrollan y complementan las previsiones contenidas en el estudio o estudio básico, en función sistema de ejecución de la obra de la empresa, lo que permitirá su control económico posterior.

Por su parte, el ESS forma parte del proyecto de ejecución de la obra, recoge las medidas preventivas adecuadas a los riesgos que conlleve la realización de la obra. El presupuesto de dicho ESS es la valoración económica del conjunto de gastos previstos para la aplicación y ejecución de todas las medidas preventivas recogidas en el estudio de seguridad y salud. Es en esta fase, de diseño, donde deben focalizarse los esfuerzos en el análisis de las medidas de prevención necesarias para la posterior fase de ejecución (13).

Ambos documentos tienen su origen en la Directiva 92/57/ CEE del Consejo de 24 de junio de 1992 relativa a las disposiciones mínimas de seguridad y salud que deben aplicarse en las obras de construcción temporales o móviles, donde realmente tienen su origen dichos documentos (14), en el que además se recoge que en ningún caso el presupuesto del PSS será menor que el del ESS.

\subsection{Costes de la siniestralidad}

Antes de analizar los costes que supone la siniestralidad, se definen algunos términos que posteriormente nos ayudarán al análisis de los mismos.

Se define siniestro como el daño de cualquier importancia que puede ser indemnizado por una compañía aseguradora (15). Así mismo, se define siniestralidad como la frecuencia o índice de siniestros. Si unimos a esta definición, la definición de laboral, perteneciente o relativo al trabajo, en su aspecto económico, jurídico y social, convenimos en que en prevención de riesgos laborales la siniestralidad laboral se refiere a la frecuencia con la que se producen daños de distinto índole relacionados con el desarrollo del trabajo.

El término siniestralidad laboral recoge dos conceptos: accidentes de trabajo y enfermedades profesionales. En el primer caso nos encontramos ante un efecto directo, claramente identificable, mientras que en el segundo caso la relación causal entre condiciones de trabajo y enfermedad puede ser, en muchas ocasiones, de naturaleza indirecta o producirse con retraso, después de un determinado periodo de exposición al riesgo o al agente causante ${ }^{1}(16)$.

${ }^{1}$ Esta investigación se enfoca únicamente al caso de los accidentes de trabajo. 
Se delimitan los costes tangibles de la siniestralidad como aquellos costes que se derivan de la ocurrencia de un accidente en la empresa, y cuya expresión cuantitativa puede realizarse recurriendo a la metodología de cálculo tradicional. Por tanto, recogen los costes correspondientes a accidentes tales como:

- accidentes con lesiones, con o sin baja;

- accidentes que produzcan pequeñas lesiones (pequeños cortes, contusiones rasguños, etc.), que se solucionen con una cura de botiquín;

- accidentes que, sin haber causado lesiones de ningún tipo, sean potenciales de causarlas, si se vuelve a repetir el suceso que los ha provocado;

- accidentes solo con daños materiales;

- accidentes que impliquen un paro significativo en el proceso productivo;

- incidentes que supongan un tiempo perdido significativo;

- así como el coste de las enfermedades profesionales.

Por su parte, los costes intangibles de la siniestralidad, siguiendo a Gosellin, son aquellos costes que no son medibles en términos económicos o de los que no se dispone de índices de funcionamiento capaces de medir su repercusión en la organización (5), como la baja moral de los trabajadores o la pérdida de mercado, y que por su naturaleza, no se han estimado en esta investigación.

\subsection{Costes de Prevención}

Fruto de esta investigación, se define el término «costes de prevención» como los costes que la empresa asume para dar cumplimiento legal a los requerimientos empresariales en materia preventiva, el coste de todas las medidas necesarias para la implementación de las medidas de prevención de riesgos laborales en las obras de construcción y el coste de las medidas necesarias para mejorar las condiciones de seguridad y salud en los distintos ámbitos del trabajo que se desarrolla.

\section{METODOLOGÍA}

El abanico de herramientas para llevar a cabo una investigación es muy amplio. La Comisión Europea y la Oficina de Cooperación EuropeAid, ha clasificado trece herramientas que pueden ser utilizadas para la evaluación de proyectos, programas e intervenciones (17). Para seleccionar la herramienta que mejor optimiza los resultados de esta investigación, tras la revisión bibliográfica realizada y teniendo en cuenta las recomendaciones europeas, se ha desarrollado una metodología para analizar la inversión en PRL, la siniestralidad y sus costes, que consta de dos fases.

En una primera, en la que se aplica metodología cualitativa, cuyo objetivo es el diseño del cuestionario para comprobar si se ha alcanzado el objetivo, se establecen unas hipótesis apriorísticas y el modo de plasmarlas en un cuestionario inicial se somete al panel de expertos. Una vez consensuado en el panel de expertos se redactó el Cuestionario o. A modo de pre test, se envió a tres obras civiles que estaban en fase de ejecución, todas en Andalucía, durante el período 20062007. Una vez cumplimentado por los responsables de las mismas (ya fuese jefe de obra o técnico de prevención...), se redacta a partir de él el cuestionario definitivo.

En la segunda fase la metodología es mixta (cuantitativa-cualitativa) enfocada en la implementación de la encuesta en las obras de construcción y al análisis de los resultados obtenidos. En esta etapa es fundamental el trabajo realizado in situ que facilita la descripción sistemática del escenario social en el que se desarrollan las obras de construcción. Esta metodología recoge el hecho de que en esta investigación se distinga una faceta técnica y otra faceta social. El primer hecho que se detectó fue el escaso número de datos reflejados, la dificultad de recogerlos especialmente cuando se refieren a los accidentes. Esto implicó que quedaron sin cumplimentar el resto de respuestas en relación a las características de los accidentes. A la vista de ello se decidió la participación activa de los encuestadores: observación participante en la cumplimentación del cuestionario definitivo in situ. Por otro lado, se modificó la redacción de preguntas de respuesta condicionada como, por ejemplo, las del tipo: «en caso afirmativo continuar en...».

Se detallan a continuación cada una de las herramientas utilizadas.

\subsection{La muestra}

Se hizo la selección de la muestra a partir de la Estadística de la Construcción ofrecida por el INE, año 2007, en la que figuraba un total de 395 obras en la Comunidad Autónoma Andaluza. Se trataba de obras en ejecución que podemos clasificar en los grupos siguientes: carreteras, paseos marítimos, ferrocarriles, urbanizaciones, aeropuertos, viviendas unifamiliares y aparcamientos. Esta población se dividió en estratos según el presupuesto de ejecución por contrata de dichas obras, resultando un total de 6 estratos. Dentro de cada estrato, se codificó cada obra asignándole una cifra. Las obras que pasaron a formar parte de la muestra en cada uno de los estratos fueron seleccionadas aplicando el muestreo aleatorio simple. Así quedó establecido tanto el número de obras para cada estrato como la identificación de cada una de ellas.

Se eligió una muestra con 40 obras, todas en Andalucía, con objeto de abarcar las facetas más importantes del mercado de la Construcción, la tipología de las obras elegidas se divide entre obra civil y edificación. Cada una de ellas se encontraba en distintas etapas del proceso productivo, lo cual permitía analizar el grado de implantación de las medidas de Seguridad y Salud y el coste que estas generan. Se analizaron un total de 178 accidentes, se entrevistó a más de 200 trabajadores intervinientes en el proceso productivo relacionados con la prevención, como jefes de obras o coordinadores de seguridad y salud.

\subsection{Metodología cuantitativa. El cuestionario.}

En la fase de trabajo de campo de la investigación se recoge la información de las obras mediante la aplicación de un cuestionario (18) (19). El cuestionario, como herramienta de medida, permite obtener datos de una determinada realidad social.

El cuestionario se desarrolla en preguntas de distinto tipo (20), que se han agrupado en bloques o áreas temáticas atendiendo al contenido de la información que nos proponemos obtener, datos generales (tipología de construcción, presupuesto de ejecución, baja, plazo de ejecución, importe del plan de seguridad y salud...), costes (protecciones colectivas y fase en la que se aplican, equipos de protección individual, brigada de seguridad....), trabajadores (número de trabajadores propios, número de empresas subcontratistas, ...) y 
siniestralidad (número de accidentes, fase en la que ocurre el accidente, duración de la baja...). Dentro de cada área temática, se parte de preguntas más generales para continuar con otras más concretas y, en todo momento, se ha tratado de evitar que el encuestado tenga que seguir indicaciones complejas, para darles respuesta.

\subsection{Metodología cualitativa. El panel de expertos y la observación participante}

El empleo de procedimientos cuantitativos y cualitativos en una investigación intenta evitar los sesgos propios de cada método, pero como afirma Pita Fernández (21) la metodología cuantitativa es la más empleada por la evolución del método científico a lo largo de los años. En la actualidad, sin embargo, son cada vez más necesarios los enfoques cualitativos, ya que nos conducen y nos ayudan a comprender fenómenos sociales en el medio natural en el que se desarrollan, como las obras de construcción, mientras que la metodología cuantitativa se centra en enumerar en circunstancias concretas pruebas controladas aleatoriamente.

En los datos de costes de seguridad y salud, como en el caso de los datos de siniestralidad donde es manifiesta la falta de datos de los accidentes (22), existe otra dificultad añadida en la cuantificación, que surge por la peculiaridad en la recogida de los datos correspondientes, ya que como destaca Laufer (23) esta puede producirse:

- en distintos momentos de tiempo: cuando ocurre el accidente, cuando se sustituye al trabajador o cuando se incorpora el trabajador recuperado;

- en distintos espacios: en el lugar del accidente, en el centro de atención médica o en los departamentos administrativos de la empresa;

- por diferentes organizaciones: la propia empresa, la Seguridad Social o las compañías de seguro.

Es por ello que a pesar de que una de las limitaciones de los métodos cualitativos es su dificultad para generalizar (24), se decide implementar en esta investigación herramientas cualitativas, en este caso con un panel de expertos y con la observación participante. El panel de expertos se introduce, porque através de la opinión de éstos, más subjetivas y en consecuencia menos medibles, permite adaptarse a una realidad más dinámica como la representada por los aspectos relacionados con la seguridad y salud. Algunas experiencias pueden encontrarse en los trabajos de Love (25), Hallowell \& Gambatesse (22) o Andriessen (26). Mediante la observación, se recogen datos de personas, procesos y culturas en investigaciones cualitativas, que permite la descripción sistemática en un escenario social (como las obras de la muestra).

\subsubsection{Panel de expertos}

El panel de expertos es un grupo de especialistas independientes y reputados en, al menos, uno de los aspectos concernidos por el aspecto que se va a evaluar, al que se reúne para que emita un juicio colectivo y consensuado sobre dicho aspecto (27). En esta investigación el panel de expertos está formado por once personas, entre los cuales están representados el ámbito empresarial y económico de las obras de construcción, la gestión y la dirección de la prevención de riesgos laborales de las empresas, la administración laboral, y el ámbito universitario
Una vez seleccionadas las personas más indicadas (expertos) se somete a su juicio el cuestionario. Tras el consenso del panel de expertos, con los resultados aportados, se modifica aquel, obteniéndose el cuestionario o que será utilizado en la prueba piloto. Finalmente se obtiene el cuestionario mejorado (cuestionario inicial) una vez subsanados los problemas detectados en la prueba piloto, es decir, una vez eliminadas las preguntas con respuesta condicionada y decidido el apoyo a los empresarios en la cumplimentación del cuestionario.

\subsubsection{La observación participante}

Para poder comprender los fenómenos en estudio de manera que este sea objetivo y preciso, la observación participante, posibilita recoger diferentes datos, reducir la actitud de actuar de la gente al ser observada, desarrollar la formulación de preguntas con sentido, dar comprensión y credibilidad y finalmente recoger datos de forma óptima.

Sin embargo presenta algunas limitaciones como que se realizan diferentes observaciones en función del investigador que las realice, de ahí que se optara por la participación de investigadora principal en toda la recogida de datos, es decir, el observador como participante.

\subsection{El trabajo de campo}

Una vez que se habían identificado las 40 obras resultantes tras la realización del muestreo aleatorio estratificado descrito anteriormente, se procedió a ajustarlo a la realidad consultando las obras que estaban en fase de ejecución en la base de datos de obras de Andalucía, eliminando de la lista teórica aquellas en las que en ese momento habían cambiado sus condiciones y sustituyéndolas por otras equivalentes. Se corroboró que la ejecución se encontraba en curso mediante la realización de llamadas telefónicas a las obras previamente seleccionadas. Una vez seleccionadas las obras de la muestra, se contactó con el Jefe de obra de cada una de ellas para explicarle el objeto de esta investigación y garantizarle la confidencialidad del estudio. Posteriormente, se elaboró el programa de trabajo y de visitas a las obras, consensuado con el personal de cada una de ellas. Se programaron un mínimo de dos visitas a cada una de las obras, salvo en aquellas en las que el Grado de Avance estuviese cercano al 100\% para las que se programó una única visita.

Posteriormente, dos investigadores se desplazaron a las obras de la muestra seleccionada. En la primera visita se realizó una presentación de la investigación, en la que se explicó el objetivo de la misma y su desarrollo: fechas de las visitas, plazos de la investigación y documentación a consultar. En esta primera visita se convocó al jefe de obra, a los encargados, al técnico de Prevención de la empresa contratista, al administrativo de obra y al coordinador de Seguridad y Salud en la fase de construcción. Tras esta reunión, una vez comprobada la fase de construcción en la que se encontraba y el grado de implementación de las medidas de prevención previstas, se procedía a la cumplimentación del cuestionario, a la vez que se recogía toda la documentación complementaria al mismo. Posteriormente, entre 30 y 60 días después de la primera visita, se realizaba la segunda visita, para completar la documentación que no se había podido recabar en la primera, o comprobar si había habido alguna nueva circunstancia susceptible de reflejar en el cuestionario (accidentes, visitas de inspección...). 


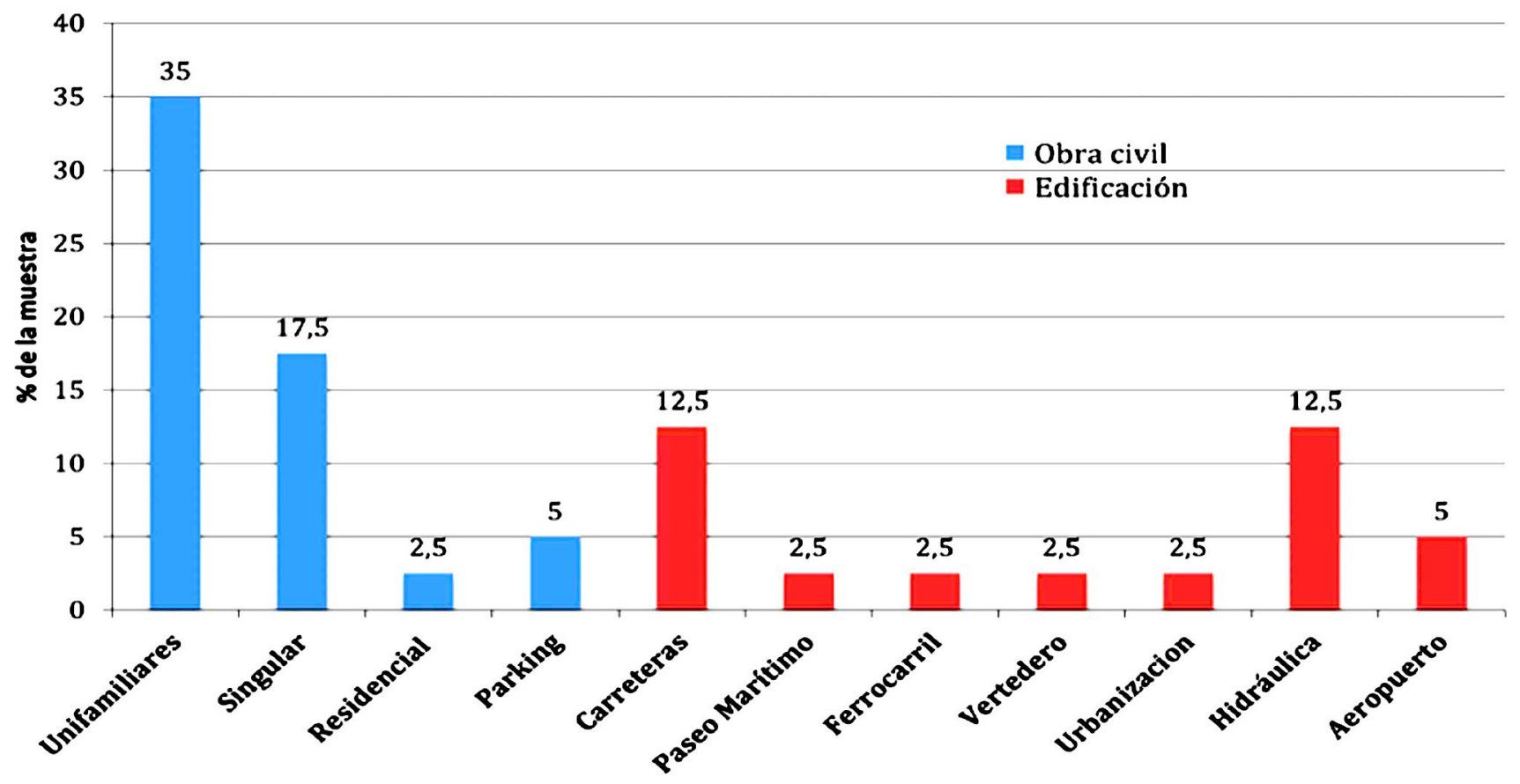

Figura 1. Distribución de obras según tipologías. Fuente: Elaboración propia.

Posteriormente se digitalizó la información recabada en la base de datos del programa informático SPSS IBM 15.0 para Windows se realizó el tratamiento de los mismos con STATA. En ocasiones, durante el desarrollo de esta fase, ha sido preciso volver a visitar la obra objeto de estudio para corregir errores del cuestionario o, simplemente, para completar algún ítem del mismo.

\subsection{El análisis estadístico}

En primer lugar, definimos las variables de estudio como cualquier característica, propiedad o cualidad de una población, que puede variar o cambiar y que afectan a nuestra investigación. En esta investigación se han definido un total de 24 variables, alguna de ellas se obtienen directamente del cuestionario (D), otras se calculan a partir de los datos recogidos del cuestionario (C) y la variable coste de los accidentes estimada (E) según la fórmula de trabajo del método de cálculo de costes de los accidentes aportada por el Instituto Navarro de Seguridad Laboral (28), basada en la Nota Técnica de Prevención 540, del Instituto Nacional de Seguridad e Higiene en el Trabajo (29), que ha servido de referencia para diversos estudios realizados en España, a nivel agregado, como el desarrollado por el Centro Nacional de Condiciones de Trabajo del INSHT en el año 2001, el realizado por el Instituto Riojano de Salud Laboral (30) o el correspondiente al Instituto Vasco de Salud Laboral (31).

Las principales variables utilizadas son: emplazamiento de las obras, tipología de obra, presupuesto de ejecución material y por contrata, plazo de ejecución, grado de avance, baja de adjudicación, tipo de contratación, accidentalidad, costes de las medidas de prevención y coste de los accidentes.

Mediante el contraste de las hipótesis, se observan las relaciones existentes entre las variables del estudio empírico, especialmente las relacionadas con el coste. Al hacer la prueba de normalidad de los datos con el test de Kolmogorov (32), se comprobó que las variables no seguían una distribución normal a excepción de la variable media de trabajadores propios que si se adaptaba a una distribución normal, por tanto, se utilizó en el análisis bivariante tests no paramétricos, excepto en los contrastes en los que interviniera la variable anterior.

Son varios los test no paramétricos que podemos aplicar con los condicionantes de nuestra muestra. Se utilizan los coeficientes Rho de Spearman y el coeficiente de Pearson, que son descriptivos estadísticos que miden el grado en que las variables se relacionan (33).

\section{RESULTADOS Y DISCUSIÓN}

A continuación se describen las características de la muestra de estudio, de las variables analizadas en la investigación y de los datos recogidos a través de la encuesta realizada en cada de una de las 40 obras que forman la muestra. Así mismo, se ofrecen los resultados obtenidos y el análisis de las relaciones entre las variables más relevantes.

\subsection{Análisis descriptivo}

El análisis descriptivo de las variables de la muestra pone de manifiesto las siguientes características: el $45 \%$ de las obras estudiadas son de promoción privada, íntegramente de edificación, y el $55 \%$ restante de promoción pública, con un $72 \%$ de obras civiles y el resto de edificación. Además, el $40 \%$ de las obras de la muestra corresponde a obra civil y el 60\% restante a obras de edificación (Figura 1). Resulta destacable en la muestra que el $35 \%$ corresponde a viviendas unifamiliares.

En cuanto al plazo de ejecución, el 47,5\% de la obras de la muestra tiene un plazo de ejecución contractual comprendido entre 18 y 24 meses, y el $60 \%$ de las obras se encuentran próximas a su finalización, con más del $70 \%$ de la producción total, ya ejecutada. 


\subsection{Presupuesto de seguridad, coste de prevención y coste de accidentes}

El importe del capítulo Plan de Seguridad y Salud (PSS), medido en valor absoluto, varía entre 10 mil y 100 mil euros en el $50 \%$ de las obras. La relación PSS/PEC (Presupuesto de Ejecución por Contrata), adquiere un valor medio para la muestra de un $1,18 \%$, por lo que, en la muestra, el valor del medio del PSS se encuentra entre el $1 \%$ y el $2 \%$ del PEC, como ocurre en general en las obras de construcción. Sin embargo, en el análisis pormenorizado de cada obra esta relación no se mantiene en todos los casos, ya que presenta un valor mínimo de $0,31 \%$ en una obra de carreteras y un máximo de un $4,38 \%$ para una obra de urbanización.

Las dos variables económicas siguientes, coste de prevención y coste de accidentes, son fruto del trabajo de campo realizado, y ha sido necesario calcularlos a partir de la información recabada en las obras, a través de la encuesta.

El coste de prevención (CP), se corresponde con el coste total de la implantación de las medidas de seguridad y salud en cada una de las obras, obtenido de los datos solicitados en el cuestionario, y se ha calculado añadiendo al coste de compra de material de prevención de riesgos, el coste a la brigada de seguridad o el correspondiente a la subcontrata, en su caso, que implanta las medidas de seguridad, así como el coste de compra de los equipos de protección individual. En la muestra, el coste total de prevención asciende a 4.270.722,78 €, repartidos en distintas fases de las obras y en distintos elementos necesarios para la mejora de las condiciones de seguridad y salud o, simplemente, como cumplimiento del plan de seguridad y salud.

Como se observa en la Figura 2, el 32,13\% del coste de prevención total de la muestra corresponde al coste de las medidas de seguridad y salud adoptadas en la fase de estructuras, seguido del coste de prevención en la fase de movimiento de tierras con un $18,39 \%$. De este modo, los mayores costes de prevención se producen en las fases de mayor siniestralidad de la muestra, como se muestra en la Tabla 1, corroborándose así que en las fases en las que se realiza la mayor inversión en

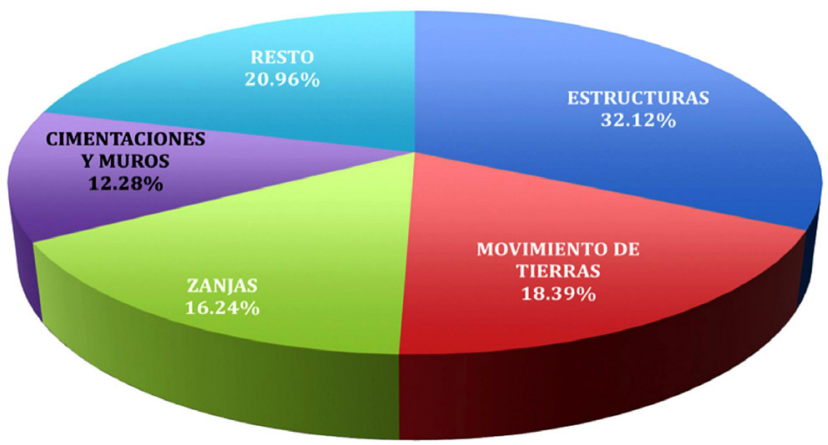

Figura 2. Distribución del coste total de Prevención por fases de obra Fuente: Elaboración propia.

medidas de prevención son aquellas en las que resulta mayor el riesgo de accidente.

Además, de los resultados de la encuesta se desprende que aquellas obras con más de un $80 \%$ de la producción ejecutada, el CP supera al del PSS en un $77 \%$ de las mismas, lo cual pone de manifiesto que, en nuestra muestra, las medidas de seguridad previstas y recogidas en PSS no se corresponden con las que en la ejecución son realmente necesarias y, por tanto, se hace necesario un estudio exhaustivo y pormenorizado de las medidas de prevención al inicio de las obras y durante la ejecución de las mismas, ya que en las obras de la muestra se ha gastado más de lo previsto (ver Tabla 2).

Por otro lado, el conocimiento del uso y vida útil que pudieran tener dichas medidas de protección, en caso de ser utilizadas en más de una obra, permitiría calcular su coste de amortización, y proceder así a un reparto equitativo de su coste entre las distintas obras.

En el análisis bivariante llevado a cabo, cuando se plantea la relación entre grado de avance y coste de prevención se obtiene que no existe correlación significativa, es decir, el coste de la implantación de medidas de seguridad es independiente del grado de avance. En la muestra se comprueba que el coste de prevención evoluciona linealmente hasta el $50 \%$ de avance, donde se mantiene constante y repunta al final. De

Tabla 1. Distribución de accidentes según fase de ejecución. Fuente: Elaboración propia.

\begin{tabular}{|c|c|c|c|c|c|}
\hline \multirow[b]{2}{*}{ Fase de Ejecución } & \multicolumn{2}{|c|}{$\begin{array}{c}\mathrm{N}^{\circ} \text { de accidentados de personal } \\
\text { propio }\end{array}$} & \multicolumn{2}{|c|}{$\begin{array}{c}\text { No de accidentados de personal de } \\
\text { empresas subcontratistas }\end{array}$} & \multirow{2}{*}{$\begin{array}{c}\text { Total } \\
\text { Accidentados } \\
\text { por fase }\end{array}$} \\
\hline & $\begin{array}{l}\mathrm{N}^{\circ} \text { de } \\
\text { accidentados }\end{array}$ & $\begin{array}{l}\text { \% sobre el total } \\
\text { de accidentados } \\
\text { en cada fase }\end{array}$ & $\begin{array}{c}\mathrm{N}^{\circ} \text { de } \\
\text { accidentados }\end{array}$ & $\begin{array}{l}\text { \% sobre el total } \\
\text { de accidentados } \\
\text { en cada fase }\end{array}$ & \\
\hline Estructuras & 35 & $36,09 \%$ & 62 & $63,90 \%$ & 97 \\
\hline Movimiento de tierras & 22 & $88,00 \%$ & 3 & $12,00 \%$ & 25 \\
\hline Acabados & 5 & $45,45 \%$ & 6 & $54,54 \%$ & 11 \\
\hline Instalaciones & 2 & $18,18 \%$ & 9 & $81,81 \%$ & 11 \\
\hline Cerramientos & 6 & $66,66 \%$ & 3 & $33,33 \%$ & 9 \\
\hline Urbanización & 4 & $57,14 \%$ & 3 & $42,85 \%$ & 7 \\
\hline Drenaje & 3 & $50,00 \%$ & 3 & $50,00 \%$ & 6 \\
\hline Cimentación & 0 & $0,00 \%$ & 3 & $0,00 \%$ & 3 \\
\hline Aglomerados & 2 & $100,00 \%$ & $\mathrm{O}$ & $0,00 \%$ & 2 \\
\hline Gruistas & 2 & $100,00 \%$ & $\mathrm{O}$ & $0,00 \%$ & 2 \\
\hline Replanteo & 2 & $100,00 \%$ & $\mathrm{O}$ & $0,00 \%$ & 2 \\
\hline Zanjas & 2 & $100,00 \%$ & $\mathrm{O}$ & $0,00 \%$ & 2 \\
\hline Otros & 1 & $100,00 \%$ & $\mathrm{O}$ & $0,00 \%$ & 1 \\
\hline TOTAL ACCIDENTADOS & 86 & $48,32 \%$ & 92 & $51,68 \%$ & 178 \\
\hline
\end{tabular}


Tabla 2. \% Coste real de la Prevención frente al importe del PSS. Fuente: Elaboración propia.

\begin{tabular}{|r|c|c|c|c|}
\hline ID & $\begin{array}{c}\text { GRADO } \\
\text { AVANCE (\%) }\end{array}$ & PSS & CP & $\begin{array}{c}\text { \% (CP- } \\
\text { PSS)/PSS }\end{array}$ \\
\hline 33 & 84,7 & 9353,66 & 3774,2 & $-59,65$ \\
\hline 8 & 83,93 & 609578,45 & 316278,5 & $-48,12$ \\
\hline 34 & 89,17 & 79427,62 & 57667,54 & $-27,40$ \\
\hline 5 & 98,05 & 108781,03 & 91083,96 & $-16,27$ \\
\hline 26 & 92,81 & 56500 & 53688,31 & $-4,98$ \\
\hline 38 & 100 & 38082 & 38082 & 0,00 \\
\hline 24 & 90,42 & 147665 & 155651,29 & 5,41 \\
\hline 36 & 100 & 20193,37 & 21760,99 & 7,76 \\
\hline 31 & 100 & 35824,42 & 39037,17 & 8,97 \\
\hline 22 & 90 & 135638,18 & 155497,69 & 14,64 \\
\hline 14 & 100 & 11429,32 & 13465,36 & 17,81 \\
\hline 13 & 100 & 21817,85 & 26158,36 & 19,89 \\
\hline 15 & 100 & 50043,96 & 61417,84 & 22,73 \\
\hline 20 & 95 & 80031,79 & 99648,46 & 24,51 \\
\hline 37 & 100 & 24001 & 30661,77 & 27,75 \\
\hline 18 & 100 & 52910,75 & 68248,38 & 28,99 \\
\hline 10 & 100 & 76191,88 & 110067,13 & 44,46 \\
\hline 23 & 100 & 108874,45 & 175582,14 & 61,27 \\
\hline 12 & 100 & 81297,29 & 143010,42 & 75,91 \\
\hline 11 & 100 & 61023,8 & 108561,92 & 77,90 \\
\hline 32 & 100 & 12713,02 & 37700,61 & 196,55 \\
\hline 39 & 98,71 & 91002,98 & 350901,72 & 285,59 \\
\hline
\end{tabular}

ello, se desprende que, a pesar de la planificación preventiva, las medidas de seguridad y salud se implantan según otras necesidades que aparecen durante la ejecución.

En relación al coste de los accidentes, se ha estimado el valor de los 178 accidentes ocurridos en la muestra de estudio, de acuerdo con la metodología aportada por el Instituto Nacional de Seguridad e Higiene en el Trabajo (en adelante INSHT) en su nota técnica de prevención (NTP) 540, Costes de los accidentes de trabajo: procedimiento de evaluación (29). Ha sido necesario recurrir a esta estimación, puesto que en las obras encuestadas no existía ningún registro de los datos económicos sobre el coste de los accidentes que habían ocurrido.

Del análisis comparativo de las variables coste de prevención y coste de accidente se obtiene que, aún cuando en un nú- mero elevado de obras se observa que la existencia de coste de prevención coincide con un coste de accidente nulo, en otras ocasiones, las obras que soportan coste de prevención soportan también coste de accidente. De este modo, se constata que la implantación de medidas de seguridad mejoran las condiciones de trabajo y cumple con los compromisos empresariales en materia de seguridad y salud, pero no evita la ocurrencia de los accidentes. Además, en caso de ocurrencia de un accidente, éste acarreará un coste.

Los accidentes de trabajo se han analizado tanto en valor absoluto como con ratios comparativos con otras variables, tales como grado de avance (\% de producción ejecutada con respecto a la producción total), plazo de ejecución, tipología de contratación o fases de ejecución.

En relación al grado de avance, el 46,07\% de los accidentes se concentran entre el 30 y el $60 \%$ de la obra. Al analizarlo por fase de ejecución, el 54,49\% de los accidentes totales, se producen en la fase de estructura, es decir, en actividades que se realizaban durante la ejecución de la estructura; el 14,04\% en movimiento de tierras y el resto se reparte entre las demás fases, según se muestra en la Tabla 1, donde se ordenan las fases de mayor a menor siniestralidad.

Por su parte, el tipo de contrato y su influencia en la siniestralidad ha sido puesto de manifiesto en múltiples ocasiones y por diversos autores. En la muestra objeto de estudio, los 178 accidentes han afectado tanto al personal propio de las empresas seleccionadas $(48,31 \%)$, como al personal de empresas subcontratadas $(51,69 \%)$, lo que pone de manifiesto una distribución equitativa. No obstante, en la Tabla 3 se observa que el porcentaje de accidentes que afecta al personal propio es mayor en las obras civiles $(66,67 \%)$; en cambio, en edificación afecta en mayor proporción al personal de las empresas subcontratistas $(59,68 \%)$.

El análisis de los accidentes ocurridos en la muestra, con las datos obtenidos de los documentos de investigación de los mismos (34) pone de manifiesto que según tipología de obra, el 69,66\% de los accidentes se dan en obras de edificación, mientras que en obra civil se registran menos accidentes $(30,34 \%)$ que afectan mayoritariamente a personal propio (ver Tabla 3).

Si bien en la muestra globalmente considerada, se observa una distribución equitativa de los accidentes producidos entre el personal propio y el correspondiente a la empresa subcontratista, en cambio, cuando se analizan los accidentados en las distintas fases de ejecución esta distribución

Tabla 3. Distribución de accidentes según tipologías de contratación. Fuente: Elaboración propia.

\begin{tabular}{|c|c|c|c|c|c|}
\hline \multicolumn{7}{|c|}{ Total Accidentes: 178 } \\
\hline \multicolumn{7}{|c|}{ PP (Personal Propio) } & \multicolumn{2}{c|}{ PS (Personal Empresa Subcontratista) } \\
\hline \multicolumn{7}{|c|}{86} & $48,31 \%$ & \multicolumn{2}{c|}{92} & $51,69 \%$ \\
\hline Privado & 83 & $46,63 \%$ & Público & 95 & $53,37 \%$ \\
\hline PP & 51 & $61,45 \%$ & PP & 55 & $57,89 \%$ \\
\hline PS & 32 & $38,55 \%$ & PS & 40 & $42,11 \%$ \\
\hline \multicolumn{7}{|c|}{ Tipo de Obra } \\
\hline Edificacion & 124 & $69,66 \%$ & Obra Civil & 54 & $30,34 \%$ \\
\hline PP & 50 & $40,32 \%$ & PP & 36 & $66,67 \%$ \\
\hline PS & 74 & $59,68 \%$ & PS & 18 & $33,33 \%$ \\
\hline
\end{tabular}


no se mantiene equitativa. Así, al analizar las fases de mayor accidentalidad se observa que, en la fase de estructura, el número de accidentados de personal propio representa el $36,08 \%$ respecto al número total de accidentes en dicha fase, frente al $63,90 \%$ del personal perteneciente a empresa subcontratada. Por su parte, en la fase de movimiento de tierras, el $88 \%$ del total de los accidentados corresponde a personal propio, frente al $12 \%$ que representa el personal de empresas subcontratistas. Por lo que podemos afirmar, tras el análisis descriptivo llevado a cabo, la necesidad de realizar un análisis bivariante en el que se obtengan resultados en la variable respuesta (accidente o costes) en función de las otras variables descritas.

Las variables analizadas son discretas y cuantitativas y no se adaptan a una distribución normal (excepto la variable media de trabajadores propios), por lo que se han utilizado test no paramétricos. Así, en el análisis bivariante de los datos obtenidos, se han utilizado el coeficiente Rho de Spearman y el coeficiente de Pearson, con significación 0.05, que permiten medir la correlación existente entre dos variables, a excepción de aquellos contrastes en los que interviene la variable mencionada, en los que se ha podido utilizar test paramétrico.

De acuerdo con otros autores, podemos afirmar que la ocurrencia de los accidentes no depende de una única variable, (1) (35) (36) (37) (38). De modo que planteamos un análisis bivariante que nos permita, mediante el contraste de un total de las 20 hipótesis planteadas, establecer las relaciones que existen entre las variables obtenidas del cuestionario y los accidentes y/o sus costes.

De acuerdo con los resultados del análisis bivariante podemos concluir que el tamaño de las obras (medido en términos económicos, según el PEC) está relacionado con los costes de seguridad (ver Figura 3). Sin embargo, un mayor importe del plan de seguridad, no disminuye el número de accidentes ni su coste. Del mismo modo, el PEC y la baja de adjudicación no presentan asociación significativa con el número de accidentes o con su coste.

Sin embargo, según los resultados del análisis bivariante, se constata que el número de accidentes y su coste tiene relación positiva directa con los trabajadores totales en la obra, con los trabajadores pertenecientes a empresas subcontratistas y con la media de empresas subcontratistas presentes en la obra.
Finalmente, resulta destacable el resultado obtenido del estudio de la relación entre el coste de prevención y el coste de los accidentes por fases de construcción, mediante la aplicación del análisis bivariante chi-cuadrado. Este análisis pone de manifiesto que el comportamiento de las variables es distinto en las fases de mayor riesgo que en las fases en las que el riesgo, a priori, es menor. Así, se observa que en las fases de estructuras, zanjas y movimiento de tierras, es decir, aquella fases en las que se ha constatado que la siniestralidad es mayor, se asumen los mayores costes de prevención y, solo en una estas fases (estructuras), se cumple la hipótesis de que cuanto mayores son los costes de prevención, menores son los costes de la siniestralidad. En cambio, en el resto de fases, se observa que no se cumple esta hipótesis.

En definitiva, del análisis bivariante efectuado por fases se obtiene que, en las fases de estructura, instalaciones, cerramientos y urbanización, a medida que aumenta el coste de prevención, disminuye el coste de los accidentes, por lo que se cumple la hipótesis de partida. Como se observa en la Figura 4 , en estas fases se concentra el $69,66 \%$ de los accidentes totales y se soporta el $44,86 \%$ de los costes de prevención totales. En el resto de fases al aumentar el coste de prevención también aumenta el coste de los accidentes.

\section{CONCLUSIONES Y RECOMENDACIONES}

Del análisis de los resultados obtenidos en esta investigación, que tiene por objeto analizar el alcance del sistema de gestión de los costes de seguridad en las empresas de construcción, se comprueba que en las 40 obras de distintas empresas que conforman la muestra objeto de estudio no se ha establecido ningún mecanismo para la gestión y el control de costes; en ningún caso se calculan los costes de prevención, ni los de siniestralidad.

Sin embargo, a partir del análisis de los datos obtenidos del estudio empírico llevado a cabo, y de acuerdo con los resultados del análisis bivariante, podemos concluir que el tamaño de las obras (medido en términos económicos, según el PEC) está relacionado con los costes de prevención incurridos. No obstante, ante un mayor importe del plan de seguridad, no se observa una disminución del número de accidentes ni de su coste. En consecuencia, se impone la necesidad de realizar un estudio exhaustivo de las medidas preventivas adecuadas en las obras de construcción y de su evolución, no sólo durante su ejecución, sino también desde la fase de diseño, al objeto

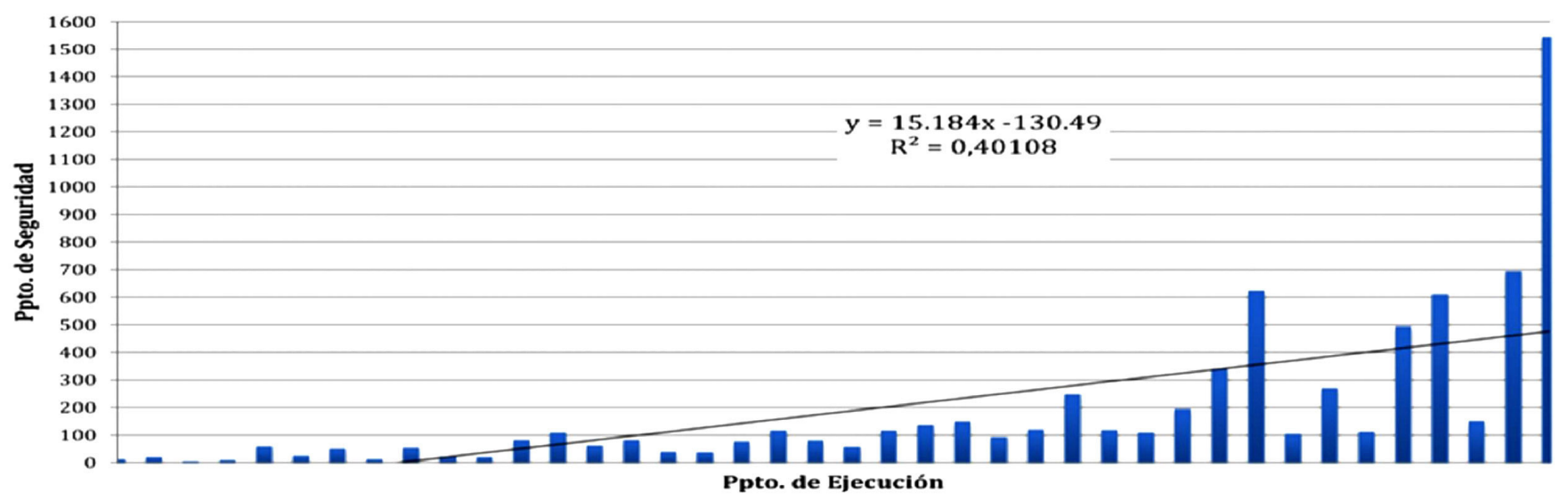

Figura 3. Relación entre las variables PSS y PEC. Fuente: Elaboración propia. 


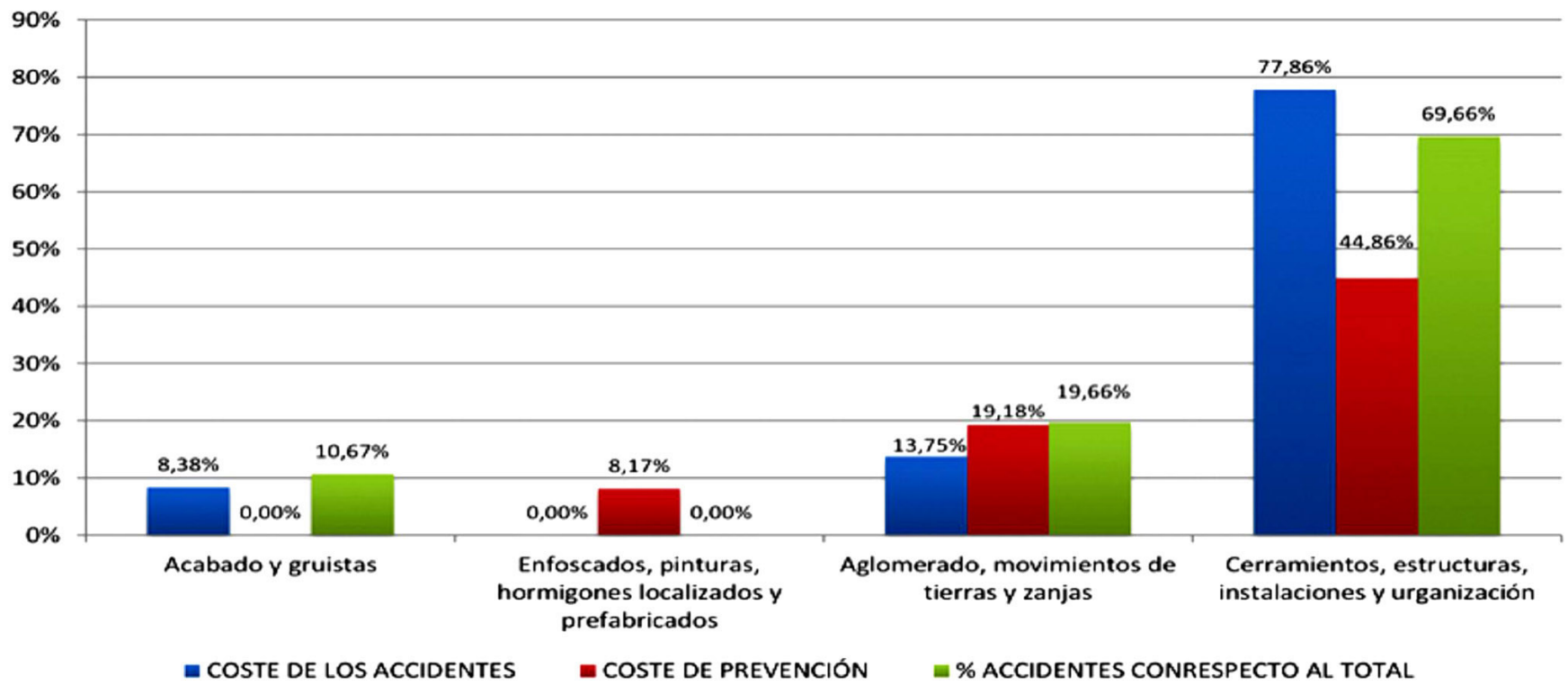

Figura 4. Comparación \% CP, \% coste de los accidentes, \% accidentes en la fase con respecto al total. Fuente: Elaboración propia

de dirigir los esfuerzos hacia aquellas fases de la obra en las que se haya detectado una mayor siniestralidad.

Por otro lado, la tipología de contratación de los trabajadores influye en los niveles de siniestralidad de las obras de construcción y en su coste. Así, un aumento en el personal perteneciente a empresas subcontratistas y/o en la media de empresas subcontratistas presentes en la obra, aumenta el número de accidentes en la misma.

Además, en las fases de obra de mayor riesgo identificadas, es decir, estructuras y movimiento de tierras, se asumen los mayores costes de prevención, a pesar de que también se obtienen los peores datos de siniestralidad. No obstante, también se ha constatado que en estas fases se cumple la hipótesis de que cuanto mayores son los costes de prevención, menores son los costes de la siniestralidad.

Finalmente, a partir de los datos empíricos recogidos en las obras de construcción y de los resultados obtenidos tras su tratamiento estadístico, comprobamos las dificultades de analizar los accidentes y sus costes en función de una única variable. En definitiva, son muchos los factores implicados en la accidentalidad que se produce en las obras de construcción y, por tanto, consideramos necesario el desarrollo de un modelo específico predictivo, que se adapte mejor a los datos relacionados con los accidentes de trabajo y sus costes. En este sentido en la segunda parte de esta investigación se ha desarrollado un modelo para la gestión de los costes de seguridad y salud, HSC_PEI2012, el cual será aplicado en obras de construcción.

\section{REFERENCIAS}

(1) Abdelhamid, T.S., Everett, J.G. (2000). Identifying root causes of construction accidents. Journal of Construction Engineering and Management, 126(1): 52-6o, doi: http://dx.doi.org/10.1061/(ASCE)0733-9364(2000)126:1(52).

(2) Hinze, J., Appelgate, L.L. (1991). Costs of construction injuries, Journal of Construction Engineering and Management, 117(3): 537-550, doi: http://dx.doi.org/10.1061/(ASCE)0733-9364(1991)117:3(537).

(3) Montero, M.J., Araque, R.A., Rey, J.M. (2009). Occupational health and safety in the framework of corporate social responsibility. Safety Science, 47(10): 1440-1445, doi: http://dx.doi.org/10.1016/j.ssci.2009.03.002.

(4) Dorman, P. (1998). Internalizing the costs of occupational injuries and illnesses: Change or chimera?, (pp. 13-35).

(5) Gosselin, M. (2004). Analyse des avantages et des coûts de la santé et de la sécurité au travail en entreprise: Développement de l'outil d'analyse, Rapport de Recherche R-375.

(6) Jallon, R., Imbeau, D., De Marcellis-Warin, N. (2011). Development of an indirect-cost calculation model suitable for workplace use. Journal of Safety Research, 42(3): 149-164, doi: http://dx.doi.org/10.1016/j.jsr.2011.05.006.

(7) Brody, B., Létourneau, Y., Poirier, A. (1990). An indirect cost theory of work accident prevention, Journal of Occupational Accidents, 13(4): 255-270, doi: http://dx.doi.org/10.1016/0376-6349(90)90033-R.

(8) Andreoni, D. (ed) (1986). The Cost of Occupational Accidents and Diseases, Occupational Safety and Health Diseases edn. Geneve: International Labour Office.

(9) Heinrich, H.W. (1930). Relation of accident statistics to industrial accident prevention. En Proceedings of the Casuallity Actuarial Society, XVI(33-34): 170-174.

(10) Leopold, E., Leonard, S. (1987). Costs of construction accidents to employers. Journal of Occupational Accidents, 8(4): 273-294, doi: http://dx.doi.org/10.1016/0376-6349(87)90004-6.

(11) Rikhardsson, P.M. (2004). Accounting for the cost of occupational accidents. Corporate Social Responsibility and Environmental Management, 11(2): 63-70, doi: http://dx.doi.org/10.1002/csr.52.

(12) Arévalo, C. (2013). Integración de la prevención en el diseño de las obras de construcción: relación con la siniestralidad laboral, análisis de su regulación normativa, bases conceptuales y desarrollo internacional. Informes de la Construcción, 65(531): 325-334, doi: http://dx.doi.org/10.3989/ic.12.006. 
(13) Vázquez, F.J., Pérez, J., Carreño, A., Callejón, A. J. (2011). Diseño de un nuevo capitel para invernaderos multitúnel. Informes de la Construcción, 63(521): 47-56, doi: http://dx.doi.org/10.3989/ic.09.009.

(14) DO. (1992). Directiva 92/57/CEE del Consejo de 24 de junio de 1992 relativa a las disposiciones mínimas de seguridad y salud que deben aplicarse en las obras de construcción temporales o móviles. Diario Oficial de las Comunidades Europeas, $\mathrm{n}^{\circ} \mathrm{L} 245$, pp. 6-22.

(15) Real Academia Española. (2012). Diccionario de la lengua española. www.rae.es.

(16) Ministerio de Trabajo y Seguridad Social. (1994). Real Decreto legislativo 1/1994, de 20 de junio, por el que se aprueba el texto refundido de la Ley General de la Seguridad Social. Boletín Oficial del Estado, no 154, pp. 20658-20708. Madrid.

(17) Martínez-Aires, M.D., Rubio-Gámez, M.C., Gibb, A. (2010). Prevention through design: The effect of European Directives on construction workplace accidents. Safety Science, 48(2): 248-258, doi: http://dx.doi.org/10.1016/j.ssci.2009.09.004.

(18) García-Ferrando, M., Ibáñez, J., Alira, F. (eds). (1986). La encuesta. Madrid.

(19) Amerigo Cuervo-Arango, M. (1993). Boletín de ANABAD. Madrid: ANABAD.

(20) Ander-Egg, E. (ed). (1977). Introducción a las técnicas de investigación social. Buenos Aires: Humanitas ed.

(21) Pita-Fernández, S., Pértegas-Díaz, S. (2002). Investigación cuantitativa y cualitativa. CAD ATEN PRIMARIA, 9: 76-78.

(22) Hallowell, M.R., Gambatese, J.A. (2010). Population and initial validation of a formal model for construction safety risk management. Journal of Construction Engineering and Management, 136(9): 981-990, doi: http://dx.doi.org/10.1061/ (ASCE)CO.1943-7862.0000204.

(23) Laufer, A. (1987). Construction Accident Cost and Management Safety Motivation. Journal of Occupational Accidents, 8(4): 295-315, doi: http://dx.doi.org/10.1016/0376-6349(87)90005-8.

(24) Ander-Egg, E. (ed) (1977). Introduccion a las técnicas de investigación social. Buenos Aires: Humanitas ed.

(25) Love, P., Davis, P., Ellis, J., Cheung, S.O. (2010). Dispute causation: Identification of pathogenic influences in construction. Engineering, Construction and Architectural Management, 17(4): 404-423, doi: http://dx.doi. org/10.1108/09699981011056592.

(26) Andriessen, J.H.T.H. (1978). Safe behaviour and safety motivation. Journal of Occupational Accidents, 1(4): 363-376, doi: http://dx.doi.org/10.1016/0376-6349(78)90006-8.

(27) EuropeAid Co-operation Office. (2005). Expert panels. http://ec.europa.eu/europeaid/evaluation/methodology/.

(28) INSL. (2003). Análisis de costes de accidentes de trabajo. Pamplona, España: Instituto Navarro de Salud Laboral.

(29) Gil-Fisa, A. (1999). NTP 54O: Costes de los accidentes de trabajo: procedimiento de evaluación. Madrid, España: Instituto Nacional de Seguridad e Higiene en el Trabajo (INSHT).

(30) IRSAL. (2008). Estudio del coste económico de los accidentes de trabajo registrados en la Comunidad Autónoma de La Rioja. Logroño, España: Instituto Riojano de Salud Laboral.

(31) OSALAN. (2006). Costes de la accidentalidad laboral en Euskadi en el año 2005. Baracaldo, España: Instituto Vasco de Seguridad y Salud Laborales País Vasco.

(32) Kolmogorov, A.N. (ed). (1956). Foundations of the Theory of Probability (2nd. Edition). New York: Chelsea Publishing Company.

(33) Sheskin, D. J. (ed). (2004). Handbook Parametric and No-Parametrics Statistical Procedures (3d. edition). London: Chapman \& Hall/CRC.

(34) Pérez-Alonso, J., Carreño-Ortega, A., Vázquez-Cabrera, F.J., Callejón-Ferre, A. J. (2012). Accidents in the greenhouse-construction industry of SE Spain. Applied Ergonomics, 43(1): 69-80, doi: http://dx.doi.org/10.1016/j.apergo.2011.03.007.

(35) Gibb, A., Haslam, R., Gyi, D., Hide, S., Duff, R. (2006). What causes accidents?. En Proceedings of the Institution of Civil Engineers: Civil Engineering, 159(2):46-50 ( $\mathrm{n}^{\circ}$ especial).

(36) Gibb, A., Hide, S., Haslam, R., Hastings, S., Suraji, A., Duff, A.R., Abdelhamid, T.S., Everett, J.G. (2001). Identifying root causes of construction accidents. Journal of Construction Engineering and Management, 127(4): 348-349, doi: http:// dx.doi.org/10.1061/(ASCE)0733-9364(2001)127:4(348).

(37) Haslam, R.A., Hide, S.A., Gibb, A.G.F., Gyi, D.E., Pavitt, T., Atkinson, S., Duff, A.R. (2005). Contributing factors in construction accidents. Applied Ergonomics, 36(4): 401-415 ( ${ }^{\circ}$ especial).

(38) Camino-López, M.A., Ritzel, D.O., Fontaneda, I., González-Alcantara, O.J. (2008). Construcción industry accidents in Spain. Journal of Safety Research, 39(5): 497-507, doi: http://dx.doi.org/10.1016/j.jsr.2008.07.006. 